\title{
Krull Dimension of Tame Generalized Multicoil Algebras
}

\author{
Piotr Malicki
}

Received: 2 September 2014 / Accepted: 20 January 2015 / Published online: 7 February 2015

(C) The Author(s) 2015. This article is published with open access at Springerlink.com

\begin{abstract}
We determine the Krull dimension of the module category of finite dimensional tame generalized multicoil algebras over an algebraically closed field, which are domestic.
\end{abstract}

Keywords Krull dimension · Tame algebra · Generalized multicoil algebra ·

Auslander-Reiten component

Mathematics Subject Classification (2010) $16 \mathrm{G} 60 \cdot 16 \mathrm{G} 70 \cdot 16 \mathrm{P} 60$

\section{Introduction and the Main Results}

Let $A$ be a finite-dimensional $k$-algebra over a fixed algebraically closed field $k$. We denote by mod $A$ the category of finitely generated $A$-modules. Here, we are interested in the Krull $\operatorname{dimension} \mathrm{K}-\operatorname{dim}(\bmod A)$ of the category $\mathcal{F}=\mathcal{F}(\bmod A)$ of all finitely presented contravariant functors from $\bmod A$ into the category $\mathcal{A} b$ of abelian groups. Following [10] the Krull-Gabriel filtration $\left(\mathcal{F}_{\alpha}\right)_{\alpha}$ of $\mathcal{F}$ is defined recursively as follows: $\mathcal{F}_{-1}=0$ and $\mathcal{F}_{0}$ is the Serre subcategory of all objects of finite length in $\mathcal{F}$. In the case when $\alpha$ is an ordinal number of the form $\beta+1$ then $\mathcal{F}_{\alpha}$ is defined to be the Serre subcategory of all objects in $\mathcal{F}$ which become of finite length in $\mathcal{F} / \mathcal{F}_{\beta}$. In the case when $\alpha$ is a limit ordinal, then $\mathcal{F}_{\alpha}$ is the union of all $\mathcal{F}_{\beta}$ with $\beta<\alpha$. If there exists an ordinal $\alpha$ with $\mathcal{F}_{\alpha}=\mathcal{F}$, then the smallest ordinal with this property is called the Krull dimension of $\mathcal{F}$.

By a result of Auslander [5], we know that $\mathrm{K}-\operatorname{dim}(\bmod A)=0$ if and only if $A$ is representation-finite. Moreover, $\mathrm{K}-\operatorname{dim}(\bmod A) \operatorname{does} n o t$ exist when $A$ is wild hereditary [6], and $\mathrm{K}-\operatorname{dim}(\bmod A)=2$ when $A$ is representation-infinite tame hereditary [10]. Our interest in the Krull dimension of $\mathcal{F}$ is also motivated by the fact that the filtration $\left(\mathcal{F}_{\alpha}\right)_{\alpha}$ of

Presented by Jon F. Carlson.

P. Malicki $(\triangle)$

Faculty of Mathematics and Computer Science, Nicolaus Copernicus University,

Chopina 12/18, 87-100 Toruń, Poland

e-mail: pmalicki@mat.umk.pl 
$\mathcal{F}$ leads to a hierarchy of exact sequences in $\bmod A$, where the Auslander-Reiten sequences form the lowest level (see [10]). It is expected that the existence of $\mathrm{K}-\operatorname{dim}(\bmod A)$ implies that $A$ is domestic, that is, there is a common bound for the numbers of one-parameter families of indecomposable $A$-modules of any fixed dimension.

We would like to mention that the generalized multicoil algebras (respectively, tame generalized multicoil algebras) form a prominent class of algebras of global dimension at most 3 , containing the class of quasitilted algebras of canonical type [14, 30] (respectively, tame quasitilted algebras of canonical type), and are obtained by sophisticated gluings of concealed canonical algebras (respectively, tame concealed algebras) using admissible algebra operations (see Section 3 for details). Moreover, recently the tame generalized multicoil algebras showed to be important in describing the structure of the module category ind $\Lambda$ of an arbitrary cycle-finite algebra $\Lambda$ (see [18, Theorems 7.1 and 7.2] or [19, Theorem 1.8]). We also refer to the article [24] for the Hochschild cohomology of generalized multicoil algebras.

The following theorem is the main result of the paper.

Theorem 1.1 Let A be a tame generalized multicoil algebra. The following statements are equivalent:

(i) $\mathrm{K}-\operatorname{dim}(\bmod A)=2$.

(ii) $\mathrm{K}-\operatorname{dim}(\bmod A)$ exists.

(iii) $A$ is domestic.

In the representation theory of algebras a prominent role is played by the algebras with a separating family of components in the following sense. A family $\mathcal{C}=\left(\mathcal{C}_{i}\right)_{i \in I}$ of components of the Auslander-Reiten quiver $\Gamma_{A}$ of an algebra $A$ is called separating in $\bmod A$ if the modules in ind $A$ split into three disjoint classes $\mathcal{P}^{A}, \mathcal{C}^{A}=\mathcal{C}$ and $\mathcal{Q}^{A}$ such that:

$(\mathrm{S} 1) \mathcal{C}^{A}$ is a sincere generalized standard family of components;

$(\mathrm{S} 2) \operatorname{Hom}_{A}\left(\mathcal{Q}^{A}, \mathcal{P}^{A}\right)=0, \operatorname{Hom}_{A}\left(\mathcal{Q}^{A}, \mathcal{C}^{A}\right)=0, \operatorname{Hom}_{A}\left(\mathcal{C}^{A}, \mathcal{P}^{A}\right)=0$;

(S3) any morphism from $\mathcal{P}^{A}$ to $\mathcal{Q}^{A}$ factors through the additive category $\operatorname{add} \mathcal{C}^{A}$ of $\mathcal{C}^{A}$.

We then say that $\mathcal{C}^{A}$ separates $\mathcal{P}^{A}$ from $\mathcal{Q}^{A}$ and write $\Gamma_{A}=\mathcal{P}^{A} \cup \mathcal{C}^{A} \cup \mathcal{Q}^{A}$. We note that then $\mathcal{P}^{A}$ and $\mathcal{Q}^{A}$ are uniquely determined by $\mathcal{C}^{A}$ (see [4, (2.1)] or [26, (3.1)]). Moreover, $\mathcal{C}^{A}$ is called sincere if any simple $A$-module occurs as a composition factor of a module in $\mathcal{C}^{A}$, and generalized standard if $\operatorname{rad}^{\infty}(X, Y)=0$ for all modules $X$ and $Y$ from $\mathcal{C}^{A}$. We refer also to the survey article [23] for the structure of arbitrary algebras with separating families of Auslander-Reiten components.

Frequently, we may recover $A$ completely from the shape and categorical behaviour of the separating family $\mathcal{C}^{A}$ of components of $\Gamma_{A}$. For example, the tilted algebras $[12,26]$, or more generally double tilted algebras [25], are determined by their (separating) connecting components. Further, it was proved in [13] that the class of algebras with a separating family of stable tubes coincides with the class of concealed canonical algebras. This was extended in [21] to a characterization of algebras with a separating family of almost cyclic coherent Auslander-Reiten components. Recall that a component $\Gamma$ of an Auslander-Reiten quiver $\Gamma_{A}$ is called almost cyclic if all but finitely many modules in $\Gamma$ lie on oriented cycles contained entirely in $\Gamma$. Moreover, a component $\Gamma$ of $\Gamma_{A}$ is said to be coherent if the following two conditions are satisfied:

(C1) For each projective module $P$ in $\Gamma$ there is an infinite sectional path $P=X_{1} \rightarrow$ $X_{2} \rightarrow \cdots \rightarrow X_{i} \rightarrow X_{i+1} \rightarrow X_{i+2} \rightarrow \cdots$ (that is, $X_{i} \neq \tau_{A} X_{i+2}$ for any $i \geq 1$ ) in $\Gamma$. 
(C2) For each injective module $I$ in $\Gamma$ there is an infinite sectional path $\cdots \rightarrow Y_{j+2} \rightarrow$ $Y_{j+1} \rightarrow Y_{j} \rightarrow \cdots \rightarrow Y_{2} \rightarrow Y_{1}=I$ (that is, $Y_{j+2} \neq \tau_{A} Y_{j}$ for any $j \geq 1$ ) in $\Gamma$.

It has been proved in [21, Theorem A] that the Auslander-Reiten quiver $\Gamma_{A}$ of an algebra $A$ admits a separating family of almost cyclic coherent components if and only if $A$ is a generalized multicoil enlargement of a (possibly decomposable) concealed canonical algebra $C$. Moreover, for such an algebra $A$, we have that $A$ is triangular, gl.dim $A \leq 3$, and $\operatorname{pd}_{A} X \leq 2$ or $\operatorname{id}_{A} X \leq 2$ for any module $X$ in ind $A$ (see [21, Corollary B and Theorem E]).

As an immediate consequence of Theorem 1.1, Theorem 3.1, the definition of separating family of components of the Auslander-Reiten quiver $\Gamma_{A}$ of an algebra $A$, and [17, Theorem 1.1] we obtain the following fact.

Corollary 1.2 Let A be a tame algebra with a separating family of almost cyclic coherent Auslander-Reiten components. The following statements are equivalent:

(i) $\mathrm{K}-\operatorname{dim}(\bmod A)=2$.

(ii) $\mathrm{K}-\operatorname{dim}(\bmod A)$ exists.

(iii) $A$ is domestic.

(iv) $\left(\operatorname{rad}^{\infty}(\bmod A)\right)^{3}=0$.

\section{Preliminaries}

Throughout this paper, $k$ will denote a fixed algebraically closed field. An algebra $A$ will always mean a basic, connected (unless otherwise specified), associative finite dimensional $k$-algebra with an identity. Thus there exists a connected bound quiver $\left(Q_{A}, I_{A}\right)$ and an isomorphism $A \cong k Q_{A} / I_{A}$. Equivalently, $A \cong k Q_{A} / I_{A}$ may be considered as a $k$-linear category, of which the object class $A_{0}$ is the set of points of $Q_{A}$, and the set of morphisms $A(x, y)$ from $x$ to $y$ is the quotient of the $k$-vector space $k Q_{A}(x, y)$ of all formal linear combinations of paths in $Q_{A}$ from $x$ to $y$ by the subspace $I_{A}(x, y)=k Q_{A}(x, y) \cap I_{A}$ (see [7]). An algebra $A$ with $Q_{A}$ acyclic (without oriented cycles) is said to be triangular. A full subcategory $C$ of $A$ is said to be convex if any path in $Q_{A}$ with source and target in $Q_{C}$ lies entirely in $Q_{C}$.

By an $A$-module is meant a finitely generated right $A$-module. We denote by $\bmod A$ the category of $A$-modules, by ind $A$ the full subcategory consisting of a complete set of representatives of the isomorphism classes of indecomposable $A$-modules, by $\Gamma_{A}$ the Auslander-Reiten quiver of $A$ and by $\tau_{A}$ the Auslander-Reiten translation in $\Gamma_{A}$. We shall agree to identify the vertices of $\Gamma_{A}$ with the corresponding modules in ind $A$, and the components of $\Gamma_{A}$ with the corresponding full subcategories of ind $A$. A component $\mathcal{P}$ of $\Gamma_{A}$ is called postprojective if $\mathcal{P}$ is acyclic and every module in $\mathcal{P}$ lies in the $\tau_{A}$-orbit of a projective module. Dually, a component $\mathcal{Q}$ of $\Gamma_{A}$ is called preinjective if $\mathcal{Q}$ is acyclic and every module in $\mathcal{Q}$ lies in the $\tau_{A}$-orbit of an injective module. Recall also that the Jacobson radical $\operatorname{rad}(\bmod A)$ of the module category $\bmod A$ is the ideal of $\bmod A$ generated by all noninvertible morphisms in ind $A$. Then the infinite radical $\operatorname{rad}^{\infty}(\bmod A) \operatorname{of} \bmod A$ is the intersection of all powers $\operatorname{rad}^{i}(\bmod A), i \geq 1$, of $\operatorname{rad}(\bmod A)$.

Let $A$ be an algebra and $\mathcal{Q}$ be an infinite preinjective component of $\Gamma_{A}$. Let $\mathcal{S}$ be a set of indecomposable representatives of each infinite $\tau_{A}$-orbit of modules from $\mathcal{Q}$. Moreover, assume that for any indecomposable module $M$ from $\mathcal{S}$ there exist an indecomposable $N$ from $\mathcal{S}$ and an irreducible morphism $M \rightarrow N$ or $N \rightarrow M$. Then we say that $\mathcal{S}$ is left stable quasi-section of $\mathcal{Q}$. 
Let $A$ be an algebra and $k[x]$ the polynomial algebra in one variable. Following [9] $A$ is said to be tame if, for any dimension $d$, there exists a finite number of $k[x]-A$-bimodules $M_{i}, 1 \leq i \leq n_{d}$, which are finitely generated and free as left $k[x]$-modules, and all but a finite number of isomorphism classes of indecomposable $A$-modules of dimension $d$ are of the form $k[x] /(x-\lambda) \otimes_{k[x]} M_{i}$ for some $\lambda \in k$ and some $i \in\left\{1, \ldots, n_{d}\right\}$. Let $\mu_{A}(d)$ be the least number of $k[x]-A$-bimodules satisfying the above conditions for $d$. Then $A$ is said to be domestic if there exists a positive integer $m$ such that $\mu_{A}(d) \leq m$ for any $d \geq 1$. From the validity of the second Brauer-Thrall conjecture we know that $\mu_{A}(d)=0$ for any $d \geq 1$ if and only if $A$ is representation-finite. Recall that an algebra $A$ is said to be representationfinite if ind $A$ admits only a finite number of pairwise nonisomorphic modules. Otherwise, we say that $A$ is representation-infinite.

Let $\mathcal{C}$ be an abelian category. A full subcategory $\mathcal{C}^{\prime} \subseteq \mathcal{C}$ is called a Serre subcategory if it is closed under subobjects, quotients and extensions. If $\mathcal{C}^{\prime} \subseteq \mathcal{C}$ is a Serre subcategory, then one defines the quotient category $\mathcal{C} / \mathcal{C}^{\prime}$ as follows. The objects of $\mathcal{C} / \mathcal{C}^{\prime}$ coincide with the objects of $\mathcal{C}$, and if $X$ and $Y$ are objects of $\mathcal{C}$, then $\operatorname{Hom}_{\mathcal{C} / \mathcal{C}^{\prime}}(X, Y):=\lim _{\rightarrow} \operatorname{Hom}_{\mathcal{C}}\left(X^{\prime}, Y / Y^{\prime}\right)$, where $X^{\prime}$ and $Y^{\prime}$ run through all subobjects of $X$ and $Y$, respectively, such that $X / X^{\prime}$ and $Y^{\prime}$ belong to $\mathcal{C}^{\prime}$. Again $\mathcal{C} / \mathcal{C}^{\prime}$ is an abelian category and the quotient functor $T: \mathcal{C} \rightarrow \mathcal{C} / \mathcal{C}^{\prime}$ is exact.

Let $\mathcal{C}$ be a small abelian category. The Krull-Gabriel filtration $\left(\mathcal{C}_{\alpha}\right)_{\alpha}$ of $\mathcal{C}$ is defined as follows: $\mathcal{C}_{-1}=0, \mathcal{C}_{0}$ is the Serre subcategory of all objects of finite length in $\mathcal{C}$. In the case when $\alpha$ is an ordinal number of the form $\beta+1$ then $\mathcal{C}_{\alpha}$ is defined to be the Serre subcategory of all objects in $\mathcal{C}$ which become of finite length in $\mathcal{C} / \mathcal{C}_{\beta}$. If $\alpha$ is a limit ordinal, then $\mathcal{C}_{\alpha}$ is the union of all $\mathcal{C}_{\beta}$ with $\beta<\alpha$. If there exists an ordinal $\alpha$ with $\mathcal{C}_{\alpha}=\mathcal{C}$, then the smallest ordinal with this property is called the Krull dimension of $\mathcal{C}$, denoted by $\mathrm{K}-\operatorname{dim} \mathcal{C}$. We shall also denote by $T_{0}$ and $T_{1}$ the quotient functors $T_{0}: \mathcal{C} \rightarrow \mathcal{C} / \mathcal{C}_{0}$ and $T_{1}: \mathcal{C} \rightarrow \mathcal{C} / \mathcal{C}_{1}$, respectively.

Let $\mathcal{D}$ be a subcategory of $\bmod A$ for some algebra $A$. Denote by $\mathcal{F}(\mathcal{D})$ the category of finitely presented contravariant functors from $\mathcal{D}$ to the category $\mathcal{A} b$ of abelian groups. Assume that $\mathcal{F}(\mathcal{D})$ is abelian. Then $\mathrm{K}-\operatorname{dim} \mathcal{D}$ is by definition the Krull dimension of $\mathcal{F}(\mathcal{D})$.

The following result from [32, Lemma 2.1] will be applied.

Lemma 2.1 Let $A$ be an algebra and $\mathcal{Q}$ be an infinite preinjective component of $\Gamma_{A}$ having a left stable quasi-section $\mathcal{S}$ of Euclidean type. Assume also that any indecomposable module $M$ in $\mathcal{S}$ does not belong to a path of irreducible morphisms $N \rightarrow \cdots \rightarrow M$, where $N$ is indecomposable and the $\tau_{A}$-orbit of $N$ is finite. Then $\mathrm{K}-\operatorname{dim} \mathcal{Q}=2$.

In the proof of our main result we need also the following fact.

Lemma 2.2 Let $A$ be an algebra, $M, N, U, V$ modules in $\bmod A$, and $M \oplus U \stackrel{[f, g]}{\longrightarrow}$ $V$ and $N \stackrel{h}{\longrightarrow} U$ monomorphisms. Then the morphism $M \oplus N \stackrel{[f, g h]}{\longrightarrow} V$ is a monomorphism.

Proof The morphism $M \oplus N \stackrel{[f, g h]}{\longrightarrow} V$ is a monomorphism as the composition of the following two monomorphisms $M \oplus N \stackrel{\left[\begin{array}{cc}i d_{M} & 0 \\ 0 & h\end{array}\right]}{\longrightarrow} M \oplus U \stackrel{[f, g]}{\longrightarrow} V$.

For basic background on the representation theory of algebras applied in the paper, we refer to the books [1, 26-28]. 


\section{Tame Generalized Multicoil Algebras}

In this section we introduce and exhibit basic properties of the class of tame generalized multicoil algebras, playing the fundamental role in our proof of Theorem 1.1. This is the class of tame algebras among the class of all algebras having a separating family of almost cyclic coherent components investigated in [21,22]. Recall that a module $X$ in $\bmod A$ is called a brick if $\operatorname{End}_{A}(X) \cong k$.

It has been proved in [20, Theorem A] that a connected component $\Gamma$ of an AuslanderReiten quiver $\Gamma_{A}$ is almost cyclic and coherent if and only if $\Gamma$ is a generalized multicoil, obtained from a family of stable tubes by a sequence of operations called admissible. We recall the latter and simultaneously define the corresponding enlargements of algebras.

We start with the one-point extensions and one-point coextensions of algebras. Let $A$ be an algebra and $M$ be a module in $\bmod A$. Then the one-point extension of $A$ by $M$ is the matrix algebra

$$
A[M]=\left[\begin{array}{cc}
A & 0 \\
M & k
\end{array}\right]=\left\{\left[\begin{array}{cc}
a & 0 \\
m & \lambda
\end{array}\right] ; \lambda \in k, a \in A, m \in M\right\}
$$

with the usual addition and multiplication. The quiver $Q_{A[M]}$ of $A[M]$ contains the quiver $Q_{A}$ of $A$ as a convex subquiver, and there is an additional (extension) vertex which is a source. The $A[M]$-modules are usually identified with the triples $(V, X, \varphi)$, where $V$ is a $k$-vector space, $X$ an $A$-module and $\varphi: V \rightarrow \operatorname{Hom}_{A}(M, X)$ is a $k$-linear map. An $A[M]$ linear map $(V, X, \varphi) \rightarrow(W, Y, \psi)$ is then identified with a pair $(f, g)$, where $f: V \rightarrow W$ is $k$-linear, $g: X \rightarrow Y$ is $A$-linear and $\psi f=\operatorname{Hom}_{A}(M, g) \varphi$. Dually, one defines also the one-point coextension of $A$ by $M$ as the matrix algebra

$$
[M] A=\left[\begin{array}{cc}
k & 0 \\
D(M) & A
\end{array}\right] .
$$

For $r \geq 1$, we denote by $T_{r}(k)$ the $r \times r$-lower triangular matrix algebra

$$
\left[\begin{array}{cccccc}
k & 0 & 0 & \ldots & 0 & 0 \\
k & k & 0 & \ldots & 0 & 0 \\
k & k & k & \ldots & 0 & 0 \\
\vdots & \vdots & \vdots & \ddots & \vdots & \vdots \\
k & k & k & \ldots & k & 0 \\
k & k & k & \ldots & k & k
\end{array}\right]
$$

Given a generalized standard component $\Gamma$ of $\Gamma_{A}$, and an indecomposable module $X$ in $\Gamma$, the support $\mathcal{S}(X)$ of the functor $\left.\operatorname{Hom}_{A}(X,-)\right|_{\Gamma}$ is the $R$-linear category defined as follows [3]. Let $\mathcal{H}_{X}$ denote the full subcategory of $\Gamma$ consisting of the indecomposable modules $M$ in $\Gamma$ such that $\operatorname{Hom}_{A}(X, M) \neq 0$, and $\mathcal{I}_{X}$ denote the ideal of $\mathcal{H}_{X}$ consisting of the morphisms $f: M \rightarrow N$ (with $M, N$ in $\mathcal{H}_{X}$ ) such that $\operatorname{Hom}_{A}(X, f)=0$. We define $\mathcal{S}(X)$ to be the quotient category $\mathcal{H}_{X} / \mathcal{I}_{X}$. Following the above convention, we usually identify the $R$-linear category $\mathcal{S}(X)$ with its quiver.

From now on let $A$ be an algebra and $\Gamma$ be a family of generalized standard infinite components of $\Gamma_{A}$. For an indecomposable brick $X$ in $\Gamma$, called the pivot, one defines five admissible operations $(\mathrm{ad} 1)-(\mathrm{ad} 5)$ and their duals $\left(\mathrm{ad} 1^{*}\right)-\left(\mathrm{ad} 5^{*}\right)$ modifying the translation quiver $\Gamma=(\Gamma, \tau)$ to a new translation quiver $\left(\Gamma^{\prime}, \tau^{\prime}\right)$ and the algebra $A$ to a new algebra $A^{\prime}$, depending on the shape of the support $\mathcal{S}(X)$ (see [20, Section 2] for the figures illustrating the modified translation quivers $\Gamma^{\prime}$ ). 
(ad 1) Let $t \in \mathbb{N}$ and assume $\mathcal{S}(X)$ consists of an infinite sectional path starting at $X$ :

$$
X=X_{0} \rightarrow X_{1} \rightarrow X_{2} \rightarrow \cdots
$$

If $t \geq 1$ then $D=T_{t}(k)$ and $Y_{1}, Y_{2}, \ldots, Y_{t}$ denote the indecomposable injective $D$-modules with $Y=Y_{1}$ the unique indecomposable projective-injective $D$-module. We define the modified algebra $A^{\prime}$ of $A$ to be the one-point extension

$$
A^{\prime}=(A \times D)[X \oplus Y]
$$

and the modified translation quiver $\Gamma^{\prime}$ of $\Gamma$ to be obtained by inserting in $\Gamma$ the rectangle consisting of the modules $Z_{i j}=\left(k, X_{i} \oplus Y_{j},\left[\begin{array}{l}1 \\ 1\end{array}\right]\right)$ for $i \geq 0,1 \leq j \leq t$, and $X_{i}^{\prime}=$ $\left(k, X_{i}, 1\right)$ for $i \geq 0$. The translation $\tau^{\prime}$ of $\Gamma^{\prime}$ is defined as follows: $\tau^{\prime} Z_{i j}=Z_{i-1, j-1}$ if $i \geq 1, j \geq 2, \tau^{\prime} Z_{i 1}=X_{i-1}$ if $i \geq 1, \tau^{\prime} Z_{0 j}=Y_{j-1}$ if $j \geq 2, Z_{01}$ is projective, $\tau^{\prime} X_{0}^{\prime}=Y_{t}, \tau^{\prime} X_{i}^{\prime}=Z_{i-1, t}$ if $i \geq 1, \tau^{\prime}\left(\tau^{-1} X_{i}\right)=X_{i}^{\prime}$ provided $X_{i}$ is not an injective $A$ module, otherwise $X_{i}^{\prime}$ is injective in $\Gamma^{\prime}$. For the remaining vertices of $\Gamma^{\prime}, \tau^{\prime}$ coincides with the translation of $\Gamma$, or $\Gamma_{D}$, respectively.

Finally, if $t=0$ we define the modified algebra $A^{\prime}$ to be the one-point extension $A^{\prime}=$ $A[X]$ and the modified translation quiver $\Gamma^{\prime}$ to be the translation quiver obtained from $\Gamma$ by inserting only the sectional path consisting of the vertices $X_{i}^{\prime}, i \geq 0$.

The non-negative integer $t$ is such that the number of infinite sectional paths parallel to $X_{0} \rightarrow X_{1} \rightarrow X_{2} \rightarrow \cdots$ in the inserted rectangle equals $t+1$. We call $t$ the parameter of the operation.

Since $\Gamma$ is a generalized standard family of components of $\Gamma_{A}$, we then have that $\Gamma^{\prime}$ is a generalized standard family of components of $\Gamma_{A^{\prime}}$.

In case $\Gamma$ is a stable tube, it is clear that any module on the mouth of $\Gamma$ satisfies the condition for being a pivot for the above operation. Actually, the above operation is, in this case, the tube insertion as considered in [8].

(ad 2) Suppose that $\mathcal{S}(X)$ admits two sectional paths starting at $X$, one infinite and the other finite with at least one arrow:

$$
Y_{t} \leftarrow \cdots \leftarrow Y_{2} \leftarrow Y_{1} \leftarrow X=X_{0} \rightarrow X_{1} \rightarrow X_{2} \rightarrow \cdots
$$

where $t \geq 1$. In particular, $X$ is necessarily injective. We define the modified algebra $A^{\prime}$ of $A$ to be the one-point extension $A^{\prime}=A[X]$ and the modified translation quiver $\Gamma^{\prime}$ of $\Gamma$ to be obtained by inserting in $\Gamma$ the rectangle consisting of the modules $Z_{i j}=\left(k, X_{i} \oplus Y_{j},\left[\begin{array}{l}1 \\ 1\end{array}\right]\right)$ for $i \geq 1,1 \leq j \leq t$, and $X_{i}^{\prime}=\left(k, X_{i}, 1\right)$ for $i \geq 1$. The translation $\tau^{\prime}$ of $\Gamma^{\prime}$ is defined as follows: $X_{0}^{\prime}$ is projective-injective, $\tau^{\prime} Z_{i j}=Z_{i-1, j-1}$ if $i \geq 2, j \geq 2, \tau^{\prime} Z_{i 1}=X_{i-1}$ if $i \geq 1, \tau^{\prime} Z_{1 j}=Y_{j-1}$ if $j \geq 2, \tau^{\prime} X_{i}^{\prime}=Z_{i-1, t}$ if $i \geq 2, \tau^{\prime} X_{1}^{\prime}=Y_{t}, \tau^{\prime}\left(\tau^{-1} X_{i}\right)=X_{i}^{\prime}$ provided $X_{i}$ is not an injective $A$-module, otherwise $X_{i}^{\prime}$ is injective in $\Gamma^{\prime}$. For the remaining vertices of $\Gamma^{\prime}, \tau^{\prime}$ coincides with the translation $\tau$ of $\Gamma$.

The integer $t \geq 1$ is such that the number of infinite sectional paths parallel to $X_{0} \rightarrow$ $X_{1} \rightarrow X_{2} \rightarrow \cdots$ in the inserted rectangle equals $t+1$. We call $t$ the parameter of the operation.

Since $\Gamma$ is a generalized standard family of components of $\Gamma_{A}$, we then have that $\Gamma^{\prime}$ is a generalized standard family of components of $\Gamma_{A^{\prime}}$.

(ad 3) Assume $\mathcal{S}(X)$ is the mesh-category of two parallel sectional paths:

$$
\begin{array}{ccc}
Y_{1} & \rightarrow Y_{2} \rightarrow \cdots \rightarrow Y_{t} \\
\uparrow & \uparrow & \\
X=X_{0} & \rightarrow X_{1} \rightarrow \cdots \rightarrow X_{t-1} \rightarrow X_{t} \rightarrow \cdots
\end{array}
$$


with the upper sectional path finite and $t \geq 2$. In particular, $X_{t-1}$ is necessarily injective. Moreover, we consider the translation quiver $\bar{\Gamma}$ of $\Gamma$ obtained by deleting the arrows $Y_{i} \rightarrow$ $\tau_{A}^{-1} Y_{i-1}$. We assume that the union $\widehat{\Gamma}$ of connected components of $\bar{\Gamma}$ containing the vertices $\tau_{A}^{-1} Y_{i-1}, 2 \leq i \leq t$, is a finite translation quiver. Then $\bar{\Gamma}$ is a disjoint union of $\widehat{\Gamma}$ and a cofinite full translation subquiver $\Gamma^{*}$, containing the pivot $X$. We define the modified algebra $A^{\prime}$ of $A$ to be the one-point extension $A^{\prime}=A[X]$ and the modified translation quiver $\Gamma^{\prime}$ of $\Gamma$ to be obtained from $\Gamma^{*}$ by inserting the rectangle consisting of the modules $Z_{i j}=\left(k, X_{i} \oplus Y_{j},\left[\begin{array}{l}1 \\ 1\end{array}\right]\right)$ for $i \geq 1,1 \leq j \leq t, j \leq i$, and $X_{i}^{\prime}=\left(k, X_{i}, 1\right)$ for $i \geq 1$. The translation $\tau^{\prime}$ of $\Gamma^{\prime}$ is defined as follows: $X_{0}^{\prime}$ is projective, $\tau^{\prime} Z_{i j}=Z_{i-1, j-1}$ if $i \geq 2$, $2 \leq j \leq t, \tau^{\prime} Z_{i 1}=X_{i-1}$ if $i \geq 1, \tau^{\prime} X_{i}^{\prime}=Y_{i}$ if $1 \leq i \leq t, \tau^{\prime} X_{i}^{\prime}=Z_{i-1, t}$ if $i \geq t+1$, $\tau^{\prime} Y_{j}=X_{j-2}^{\prime}$ if $2 \leq j \leq t, \tau^{\prime}\left(\tau^{-1} X_{i}\right)=X_{i}^{\prime}$, if $i \geq t$ provided $X_{i}$ is not injective in $\Gamma$, otherwise $X_{i}^{\prime}$ is injective in $\Gamma^{\prime}$. For the remaining vertices of $\Gamma^{\prime}, \tau^{\prime}$ coincides with the translation $\tau$ of $\Gamma^{*}$. We note that $X_{t-1}^{\prime}$ is injective.

The integer $t \geq 2$ is such that the number of infinite sectional paths parallel to $X_{0} \rightarrow$ $X_{1} \rightarrow X_{2} \rightarrow \cdots$ in the inserted rectangle equals $t+1$. We call $t$ the parameter of the operation.

Since $\Gamma$ is a generalized standard family of components of $\Gamma_{A}$, we then have that $\Gamma^{\prime}$ is a generalized standard family of components of $\Gamma_{A^{\prime}}$.

(ad 4) Suppose that $\mathcal{S}(X)$ consists an infinite sectional path, starting at $X$

$$
X=X_{0} \rightarrow X_{1} \rightarrow X_{2} \rightarrow \cdots
$$

and

$$
Y=Y_{1} \rightarrow Y_{2} \rightarrow \cdots \rightarrow Y_{t}
$$

with $t \geq 1$, be a finite sectional path in $\Gamma_{A}$. Let $r \in \mathbb{N}$. Moreover, we consider the translation quiver $\overline{\bar{\Gamma}}$ of $\Gamma$ obtained by deleting the arrows $Y_{i} \rightarrow \tau_{A}^{-1} Y_{i-1}$. We assume that the union $\widehat{\Gamma}$ of connected components of $\bar{\Gamma}$ containing the vertices $\tau_{A}^{-1} Y_{i-1}, 2 \leq i \leq t$, is a finite translation quiver. Then $\bar{\Gamma}$ is a disjoint union of $\widehat{\Gamma}$ and a cofinite full translation subquiver $\Gamma^{*}$, containing the pivot $X$. For $r=0$ we define the modified algebra $A^{\prime}$ of $A$ to be the onepoint extension $A^{\prime}=A[X \oplus Y]$ and the modified translation quiver $\Gamma^{\prime}$ of $\Gamma$ to be obtained from $\Gamma^{*}$ by inserting the rectangle consisting of the modules $Z_{i j}=\left(k, X_{i} \oplus Y_{j},\left[\begin{array}{l}1 \\ 1\end{array}\right]\right)$ for $i \geq 0,1 \leq j \leq t$, and $X_{i}^{\prime}=\left(k, X_{i}, 1\right)$ for $i \geq 1$. The translation $\tau^{\prime}$ of $\Gamma^{\prime}$ is defined as follows: $\tau^{\prime} Z_{i j}=Z_{i-1, j-1}$ if $i \geq 1, j \geq 2, \tau^{\prime} Z_{i 1}=X_{i-1}$ if $i \geq 1, \tau^{\prime} Z_{0 j}=Y_{j-1}$ if $j \geq 2, Z_{01}$ is projective, $\tau^{\prime} X_{0}^{\prime}=Y_{t}, \tau^{\prime} X_{i}^{\prime}=Z_{i-1, t}$ if $i \geq 1, \tau^{\prime}\left(\tau^{-1} X_{i}\right)=X_{i}^{\prime}$ provided $X_{i}$ is not injective in $\Gamma$, otherwise $X_{i}^{\prime}$ is injective in $\Gamma^{\prime}$. For the remaining vertices of $\Gamma^{\prime}, \tau^{\prime}$ coincides with the translation of $\Gamma^{*}$.

For $r \geq 1$, let $G=T_{r}(k), U_{1, t+1}, U_{2, t+1}, \ldots, U_{r, t+1}$ denote the indecomposable projective $G$-modules, $U_{r, t+1}, U_{r, t+2}, \ldots, U_{r, t+r}$ denote the indecomposable injective $G$ modules, with $U_{r, t+1}$ the unique indecomposable projective-injective $G$-module. We define the modified algebra $A^{\prime}$ of $A$ to be the triangular matrix algebra of the form:

$$
A^{\prime}=\left[\begin{array}{cccccc}
A & 0 & 0 & \ldots & 0 & 0 \\
Y & k & 0 & \ldots & 0 & 0 \\
Y & k & k & \ldots & 0 & 0 \\
\vdots & \vdots & \vdots & \ddots & \vdots & \vdots \\
Y & k & k & \ldots & k & 0 \\
X \oplus Y & k & k & \ldots & k & k
\end{array}\right]
$$


with $r+2$ columns and rows and the modified translation quiver $\Gamma^{\prime}$ of $\Gamma$ to be obtained from $\Gamma^{*}$ by inserting the rectangles consisting of the modules $U_{s l}=\left(k, Y_{l} \oplus U_{s, t+1},\left[\begin{array}{l}1 \\ 1\end{array}\right]\right)$ for $1 \leq s \leq r, 1 \leq l \leq t$, and $Z_{i j}=\left(k, X_{i} \oplus U_{r j},\left[\begin{array}{l}1 \\ 1\end{array}\right]\right)$ for $i \geq 0,1 \leq j \leq t+r$, and $X_{i}^{\prime}=\left(k, X_{i}, 1\right)$ for $i \geq 0$. The translation $\tau^{\prime}$ of $\Gamma^{\prime}$ is defined as follows: $\tau^{\prime} Z_{i j}=Z_{i-1, j-1}$ if $i \geq 1, j \geq 2, \tau^{\prime} Z_{i 1}=X_{i-1}$ if $i \geq 1, \tau^{\prime} Z_{0 j}=U_{r, j-1}$ if $2 \leq j \leq t+r, Z_{01}, U_{k 1}, 1 \leq k \leq r$ are projective, $\tau^{\prime} U_{k l}=U_{k-1, l-1}$ if $2 \leq k \leq r, 2 \leq l \leq t+r, \tau^{\prime} U_{1 l}=Y_{l-1}$ if $2 \leq l \leq t+1$, $\tau^{\prime} X_{0}^{\prime}=U_{r, t+r}, \tau^{\prime} X_{i}^{\prime}=Z_{i-1, t+r}$ if $i \geq 1, \tau^{\prime}\left(\tau^{-1} X_{i}\right)=X_{i}^{\prime}$ provided $X_{i}$ is not injective in $\Gamma$, otherwise $X_{i}^{\prime}$ is injective in $\Gamma^{\prime}$. For the remaining vertices of $\Gamma^{\prime}, \tau^{\prime}$ coincides with the translation of $\Gamma^{*}$, or $\Gamma_{G}$, respectively.

We note that the quiver $Q_{A^{\prime}}$ of $A^{\prime}$ is obtained from the quiver of the double one-point extension $A[X][Y]$ by adding a path of length $r+1$ with source at the extension vertex of $A[X]$ and sink at the extension vertex of $A[Y]$.

The integers $t \geq 1$ and $r \geq 0$ are such that the number of infinite sectional paths parallel to $X_{0} \rightarrow X_{1} \rightarrow X_{2} \rightarrow \cdots$ in the inserted rectangles equals $t+r+1$. We call $t+r$ the parameter of the operation.

Since $\Gamma$ is a generalized standard family of components of $\Gamma_{A}$, we then have that $\Gamma^{\prime}$ is a generalized standard family of components of $\Gamma_{A^{\prime}}$.

For the definition of the next admissible operation we need also the finite versions of the admissible operations ( $\operatorname{ad} 1),(\operatorname{ad} 2),(\operatorname{ad} 3),(\operatorname{ad} 4)$, which we denote by $(\operatorname{fad} 1),(\mathrm{fad} 2)$, (fad 3$)$ and (fad 4), respectively. In order to obtain these operations we replace all infinite sectional paths of the form $X_{0} \rightarrow X_{1} \rightarrow X_{2} \rightarrow \cdots$ (in the definitions of (ad 1), (ad 2), (ad 3), (ad 4)) by the finite sectional paths of the form $X_{0} \rightarrow X_{1} \rightarrow X_{2} \rightarrow \cdots \rightarrow X_{s}$. For the operation (fad 1) $s \geq 0$, for (fad 2) and (fad 4) $s \geq 1$, and for (fad 3) $s \geq t-1$. In all above operations $X_{s}$ is injective (see [20] or [21] for the details).

(ad 5) We define the modified algebra $A^{\prime}$ of $A$ to be the iteration of the extensions described in the definitions of the admissible operations $(\operatorname{ad} 1),(\operatorname{ad} 2),(\operatorname{ad} 3),(\operatorname{ad} 4)$, and their finite versions corresponding to the operations (fad 1), (fad 2), (fad 3$)$ and (fad 4). The modified translation quiver $\Gamma^{\prime}$ of $\Gamma$ is obtained in the following three steps: first we are doing on $\Gamma$ one of the operations (fad 1), (fad 2) or (fad 3), next a finite number (possibly zero) of the operation (fad 4) and finally the operation (ad 4), and in such a way that the sectional paths starting from all the new projective vertices have a common cofinite (infinite) sectional subpath.

Since $\Gamma$ is a generalized standard family of components of $\Gamma_{A}$, we then have that $\Gamma^{\prime}$ is a generalized standard family of components of $\Gamma_{A^{\prime}}$.

Finally, together with each of the admissible operations ( $\operatorname{ad} 1),(\operatorname{ad} 2),(\operatorname{ad} 3),(\operatorname{ad} 4)$ and (ad 5), we consider its dual, denoted by $\left(\operatorname{ad} 1^{*}\right),\left(\operatorname{ad} 2^{*}\right),\left(\operatorname{ad} 3^{*}\right),\left(\operatorname{ad} 4^{*}\right)$ and $\left(\operatorname{ad} 5^{*}\right)$. These ten operations are called the admissible operations. Following [20] a connected translation quiver $\Gamma$ is said to be a generalized multicoil if $\Gamma$ can be obtained from a finite family $\mathcal{T}_{1}, \mathcal{T}_{2}, \ldots, \mathcal{T}_{s}$ of stable tubes by an iterated application of admissible operations (ad 1), $\left(\operatorname{ad} 1^{*}\right),(\operatorname{ad} 2),(\operatorname{ad} 2 *),(\operatorname{ad} 3),\left(\operatorname{ad} 3^{*}\right),(\operatorname{ad} 4),\left(\operatorname{ad} 4^{*}\right),(\operatorname{ad} 5)$ or $\left(\operatorname{ad} 5^{*}\right)$. If $s=1$, such a translation quiver $\Gamma$ is said to be a generalized coil. The admissible operations of types $(\operatorname{ad} 1),(\operatorname{ad} 2),(\operatorname{ad} 3),\left(\operatorname{ad} 1^{*}\right),\left(\operatorname{ad} 2^{*}\right)$ and $\left(\operatorname{ad} 3^{*}\right)$ have been introduced in [2-4], the admissible operations ( $\operatorname{ad} 4)$ and $\left(\operatorname{ad~} 4^{*}\right)$ for $r=0$ in [16], and the admissible operations (ad 4), $\left(\operatorname{ad~} 4^{*}\right)$ for $r \geq 1,\left(\operatorname{ad~5)}\right.$ and $\left(\operatorname{ad~} 5^{*}\right)$ in [20, 21].

Observe that any stable tube is trivially a generalized coil. A tube is a generalized coil having the property that each admissible operation in the sequence defining it is of the form $(\operatorname{ad} 1)$ or $\left(\operatorname{ad} 1^{*}\right)$. Moreover, if we apply only operations of type $(\operatorname{ad} 1)$ (respectively, of type 
$\left(\right.$ ad $\left.\left.1^{*}\right)\right)$ then such a generalized coil is a ray tube (respectively, a coray tube). Observe that a generalized coil without injective (respectively, projective) vertices is a ray tube (respectively, a coray tube). A quasi-tube is a generalized coil having the property that each of the admissible operations in the sequence defining it is of type $(\operatorname{ad} 1),\left(\operatorname{ad} 1^{*}\right),(\operatorname{ad} 2)$ or $\left(\operatorname{ad} 2^{*}\right)$. Finally, following [3] a coil is a generalized coil having the property that each of the admissible operations in the sequence defining it is one of the forms $(\operatorname{ad} 1),\left(\operatorname{ad} 1^{*}\right),(\operatorname{ad} 2),\left(\operatorname{ad} 2^{*}\right)$, $(\operatorname{ad} 3)$ or $\left(\operatorname{ad~} 3^{*}\right)$. We note that any generalized multicoil $\Gamma$ is a coherent translation quiver with trivial valuations and its cyclic part ${ }_{c} \Gamma$ (the translation subquiver of $\Gamma$ obtained by removing from $\Gamma$ all acyclic vertices and the arrows attached to them) is infinite, connected and cofinite in $\Gamma$, and so $\Gamma$ is almost cyclic.

Let $C$ be the product $C_{1} \times \ldots \times C_{m}$ of a family $C_{1}, \ldots, C_{m}$ of tame concealed algebras and $\mathcal{T}^{C}$ the disjoint union $\mathcal{T}^{C_{1}} \cup \ldots \cup \mathcal{T}^{C_{m}}$ of $\mathbb{P}_{1}(k)$-families $\mathcal{T}^{C_{1}}, \ldots, \mathcal{T}^{C_{m}}$ of pairwise orthogonal generalized standard stable tubes of $\Gamma_{C_{1}}, \ldots, \Gamma_{C_{m}}$, respectively. Following [21], we say that an algebra $A$ is a generalized multicoil enlargement of $C_{1}, \ldots, C_{m}$ if $A$ is obtained from $C$ by an iteration of admissible operations of types ( $\operatorname{ad} 1)-(\operatorname{ad} 5)$ and $\left(\operatorname{ad~} 1^{*}\right)$ (ad $5^{*}$ ) performed either on stable tubes of $\mathcal{T}^{C}$ or on generalized multicoils obtained from stable tubes of $\mathcal{T}^{C}$ by means of the operations done so far. It follows from [21, Corollary B] that then $A$ is a triangular algebra. In fact, in [21] generalized multicoil enlargements of finite families of arbitrary concealed canonical algebras (generalized multicoil algebras) have been introduced and investigated. But in the tame case we may restrict to the generalized multicoil enlargements of tame concealed algebras. Namely, we have the following consequence of [21, Theorems A and F].

Theorem 3.1 Let A be an algebra. The following statements are equivalent:

(i) A is tame and $\Gamma_{A}$ admits a separating family of almost cyclic coherent components.

(ii) $A$ is a tame generalized multicoil enlargement of a finite family of tame concealed algebras.

From now on, by a tame generalized multicoil algebra we mean a connected tame generalized multicoil enlargement of a finite family of tame concealed algebras. As a consequence of [21, Theorems $\mathrm{C}$ and $\mathrm{F}]$ and the proof of [21, Theorem $\mathrm{C}]$ we obtain the following fact.

Theorem 3.2 Let $A$ be a tame generalized multicoil algebra obtained from a family $C_{1}, \ldots, C_{m}$ of tame concealed algebras. There are full convex subcategories $A^{(l)}=$ $A_{1}^{(l)} \times \ldots \times A_{m}^{(l)}$ and $A^{(r)}=A_{1}^{(r)} \times \ldots \times A_{m}^{(r)}$ of $A$ such that the following statement hold:

(i) For each $i \in\{1, \ldots, m\}, A_{i}^{(l)}$ and $A_{i}^{(r)}$ are representation-infinite tilted algebras of Euclidean type or tubular algebras.

(ii) A can be obtained from $A^{(l)}$ by a sequence of admissible operations of types (ad 1), (ad 2), (ad 3), (ad 4) or (ad 5).

(iii) A can be obtained from $A^{(r)}$ by a sequence of admissible operations of types $\left(a d 1^{*}\right)$, $\left(\right.$ ad $\left.2^{*}\right),\left(\right.$ ad $\left.3^{*}\right),\left(\right.$ ad $\left.4^{*}\right)$ or $\left(\right.$ ad $\left.5^{*}\right)$.

(iv) The Auslander-Reiten quiver $\Gamma_{A}$ of $A$ is of the form

$$
\Gamma_{A}=\mathcal{P}^{A} \cup \mathcal{C}^{A} \cup \mathcal{Q}^{A},
$$

where $\mathcal{C}^{A}$ is a family of generalized multicoils separating $\mathcal{P}^{A}$ from $\mathcal{Q}^{A}$ such that: 
(a) $\mathcal{C}^{A}$ is obtained from the $\mathbb{P}_{1}(k)$-families $\mathcal{T}^{C_{1}}, \ldots, \mathcal{T}^{C_{m}}$ of stable tubes of $\Gamma_{C_{1}}, \ldots, \Gamma_{C_{m}}$ by admissible operations corresponding to the admissible operations leading from $C_{1}, \ldots, C_{m}$ to $A$;

(b) $\mathcal{P}^{A}$ is the disjoint union $\mathcal{P}^{A_{1}^{(l)}} \cup \ldots \cup \mathcal{P}^{A_{m}^{(l)}}$, where, for each $i \in\{1, \ldots, m\}, \mathcal{P}^{A_{i}^{(l)}}$ is either the postprojective component of $\Gamma_{A_{i}^{(l)}}$, if $A_{i}^{(l)}$ is a representation-infinite tilted algebra of Euclidean type, or $\mathcal{P}^{A_{i}^{(l)}}=\mathcal{P}_{0}^{A_{i}^{(l)}} \cup \mathcal{T}_{0}^{A_{i}^{(l)}} \cup\left(\cup_{q \in \mathbb{Q}^{+}} \mathcal{T}_{q}^{A_{i}^{(l)}}\right)$, if $A_{i}^{(l)}$ is a tubular algebra;

(c) $\mathcal{Q}^{A}$ is the disjoint union $\mathcal{Q}^{A_{1}^{(r)}} \cup \ldots \cup \mathcal{Q}^{A_{m}^{(r)}}$, where, for each $i \in\{1, \ldots, m\}, \mathcal{Q}^{A_{i}^{(r)}}$ is either the preinjective component of $\Gamma_{A_{i}^{(r)}}$, if $A_{i}^{(r)}$ is a representation-infinite tilted algebra of Euclidean type, or $\mathcal{Q}^{A_{i}^{(r)}}=\left(\bigcup_{q \in \mathbb{Q}^{+}} \mathcal{T}_{q}^{A_{i}^{(r)}}\right) \cup \mathcal{T}_{\infty}^{A_{i}^{(r)}} \cup \mathcal{Q}_{\infty}^{A_{i}^{(r)}}$, if $A_{i}^{(r)}$ is a tubular algebra.

Remark 3.3 From the proof of [21, Theorem C] we know that $A^{(l)}=A_{1}^{(l)} \times \ldots \times A_{m}^{(l)}$ (respectively, $A^{(r)}=A_{1}^{(r)} \times \ldots \times A_{m}^{(r)}$ ) is a unique maximal convex truncated branch coextension (respectively, extension) of $C=C_{1} \times C_{2} \times \cdots \times C_{m}$ inside $A$, that is, $A_{i}^{(l)}$ (respectively, $A_{i}^{(r)}$ ) is a unique maximal convex truncated branch coextension (respectively, extension) of $C_{i}$ inside $A, i \in\{1, \ldots, m\}$.

It follows from [29, Theorem 4.1] and Theorem 3.2 that, if $A$ is tame generalized multicoil algebra, then $A$ is cycle-finite (see Section 5 for the definition). Applying now [29, Theorem 5.1], we obtain the following fact.

Corollary 3.4 Let $A$ be a tame generalized multicoil algebra and $\Gamma_{A}=\mathcal{P}^{A} \cup \mathcal{C}^{A} \cup \mathcal{Q}^{A}$ the canonical decomposition of $\Gamma_{A}$. The following statements are equivalent:

(i) $A$ is domestic.

(ii) $A^{(l)}$ and $A^{(r)}$ are products of representation-infinite tilted algebras of Euclidean type.

(iii) $\mathcal{P}^{A}$ is a disjoint union of postprojective components of Euclidean type and $\mathcal{Q}^{A}$ is a disjoint union of preinjective components of Euclidean type.

\section{Proof of Theorem 1.1}

Clearly, (i) implies (ii). Let $A$ be a tame generalized multicoil algebra obtained from a family $C_{1}, \ldots, C_{m}$ of tame concealed algebras. Assume that $A$ is non-domestic. Then it follows from Theorem 3.2 (i) that there are full convex subcategories $A^{(l)}=A_{1}^{(l)} \times \ldots \times A_{m}^{(l)}$, $A^{(r)}=A_{1}^{(r)} \times \ldots \times A_{m}^{(r)}$ of $A$ and $i, j \in\{1, \ldots, m\}$ such that at least one of the algebras $A_{i}^{(l)}$ or $A_{j}^{(r)}$ is tubular. By [11, Theorem 1.6 and Proposition 4.1] the Krull dimension of a tubular algebra does not exist, and hence at least one of the dimensions $\mathrm{K}-\operatorname{dim}\left(\bmod A^{(l)}\right)$ or $\mathrm{K}-\operatorname{dim}\left(\bmod A^{(r)}\right)$ does not exist. Moreover, applying [11, Theorem 2.1], we infer that $\mathrm{K}-\operatorname{dim}(\bmod A) \operatorname{does}$ not exist too. This proves that the statement (ii) implies the statement (iii). It remains to show that (iii) implies (i). We will apply arguments similar to those applied in the proof of [32, Proposition 2.2].

By Theorem 3.2 and Remark 3.3 there exists a unique maximal convex truncated branch coextension $A^{(l)}=A_{1}^{(l)} \times \ldots \times A_{m}^{(l)}$ of $C=C_{1} \times C_{2} \times \cdots \times C_{m}$ which is a full 
convex subcategory of $A$. Moreover, $A$ is obtained from $A^{(l)}$ by a sequence of admissible operations of types (ad 1)-(ad 5). From Corollary 3.4 we know that $A^{(l)}$ is a product of representation-infinite tilted algebras $A_{1}^{(l)}, \ldots, A_{m}^{(l)}$ of Euclidean type. By [11, Theorem 3.4] we get that, for each $i \in\{1, \ldots, m\}, \mathrm{K}-\operatorname{dim}\left(\bmod A_{i}^{(l)}\right)=2$, and hence $\mathrm{K}-\operatorname{dim}\left(\bmod A^{(l)}\right)=\sup _{i=1, \ldots, m}\left\{\mathrm{~K}-\operatorname{dim}\left(\bmod A_{i}^{(l)}\right)\right\}=2$. Let $\Lambda$ be the first modified algebra of $A^{(l)}$ made to obtain $A$. Let $M$ be an indecomposable $\Lambda$-module. If $M$ belongs to a postprojective component of $\Gamma_{\Lambda}$ then $M$ belongs to the postprojective component of $\Gamma_{A_{i}^{(l)}}$ for some $i=1, \ldots, m$ and the functor $\operatorname{Hom}_{\Lambda}(-, M)$ is of finite length. Assume that $M$ belongs to a generalized multicoil $\mathcal{C}$. Since different generalized multicoils in $\bmod \Lambda$ are pairwise orthogonal, it follows from [11], that if $\mathcal{C}$ is a coray tube of $\Gamma_{A^{(l)}}$, then $T_{1} \operatorname{Hom}_{\Lambda}(-, M)=0$. Thus we may assume that $M$ is a module from the generalized multicoil of $\Gamma_{\Lambda}$ different from the coray tubes of $\Gamma_{A^{(l)}}$. If $M$ is a directing $\Lambda$-module which is not an $A^{(l)}$-module then again $\operatorname{Hom}_{\Lambda}(-, M)$ is of finite length. If $M$ is a non-directing $\Lambda$-module which is not an $A^{(l)}$-module then we have the following three cases to consider.

(a) If $\Lambda$ is a modified algebra of $A^{(l)}$ obtained by applying the admissible operation of type ( $\operatorname{ad} 1),(\operatorname{ad} 2),(\operatorname{ad} 3)$ or $(\operatorname{ad} 4)$ with $r=0$ then $M$ is isomorphic to $Z_{i j}$ or $X_{i}^{\prime}($ see Section 3). Assume first that $M \cong Z_{i j}$. Then we have an obvious monomorphism $X_{i} \oplus$ $Y_{j} \rightarrow Z_{i j}$, which induces a monomorphism of functors $\alpha: \operatorname{Hom}_{\Lambda}\left(-, X_{i} \oplus Y_{j}\right) \rightarrow$ $\operatorname{Hom}_{\Lambda}\left(-, Z_{i j}\right)$. It follows from the description of generalized multicoils that the set $\mathcal{S}_{\alpha}$ of all indecomposable modules $N$ such that $\operatorname{coker} \alpha(N) \neq 0$ is finite. Indeed, we have:

- $\quad$ For (ad 1) and (ad 4) with $r=0, \mathcal{S}_{\alpha}=\left\{Z_{k l} \mid 0 \leq k \leq i, 1 \leq l \leq j\right\}$.

- For (ad 2), $\mathcal{S}_{\alpha}=\left\{X_{0}^{\prime}, Z_{k l} \mid 1 \leq k \leq i, 1 \leq l \leq j\right\}$.

- $\quad$ For (ad 3), $\mathcal{S}_{\alpha}=\left\{X_{0}^{\prime}, \ldots, X_{j-1}^{\prime}, Z_{k l} \mid 1 \leq k \leq i, 1 \leq l \leq j, l \geq k\right\}$.

Moreover, $\operatorname{coker} \alpha$ is finitely generated. Therefore, we get that $\operatorname{coker} \alpha$ is of finite length and $T_{0} \operatorname{Hom}_{\Lambda}\left(-, X_{i} \oplus Y_{j}\right) \cong T_{0} \operatorname{Hom}_{\Lambda}\left(-, Z_{i j}\right)$. Assume now that $M \cong X_{i}^{\prime}$. Again, we have an obvious monomorphism $X_{i} \rightarrow X_{i}^{\prime}$, which induces a monomorphism of functors $\beta: \operatorname{Hom}_{\Lambda}\left(-, X_{i}\right) \rightarrow \operatorname{Hom}_{\Lambda}\left(-, X_{i}^{\prime}\right)$ and the set $\mathcal{S}_{\beta}$ of all indecomposable modules $N$ such that $\operatorname{coker} \beta(N) \neq 0$ is finite. In this subcase we get:

- For (ad 1) and (ad 4) with $r=0, \mathcal{S}_{\beta}=\left\{X_{k}^{\prime}, Z_{k l} \mid 0 \leq k \leq i, 1 \leq l \leq t\right\}$.

- For (ad 2), $\mathcal{S}_{\beta}=\left\{X_{0}^{\prime}, X_{k}^{\prime}, Z_{k l} \mid 1 \leq k \leq i, 1 \leq l \leq t\right\}$ and $\mathcal{S}_{\beta}=\left\{X_{0}^{\prime}\right\}$ when $i=0$.

Note that in the above two subcases $t$ denotes the parameter of the suitable admissible operation.

- For (ad 3), $\mathcal{S}_{\beta}=\left\{X_{0}^{\prime}, X_{k}^{\prime}, Z_{k l} \mid 1 \leq k \leq i, 1 \leq l \leq k\right\}$ and $\mathcal{S}_{\beta}=\left\{X_{0}^{\prime}\right\}$ when $i=0$.

Hence $\operatorname{coker} \beta$ is of finite length since, moreover, it is finitely generated. Thus $T_{0} \operatorname{Hom}_{\Lambda}\left(-, X_{i}\right) \cong T_{0} \operatorname{Hom}_{\Lambda}\left(-, X_{i}^{\prime}\right)$.

(b) If $\Lambda$ is a modified algebra of $A^{(l)}$ obtained by applying the admissible operation of type (ad 4) with $r \geq 1$ then $M$ is isomorphic to $U_{k l}$ for $1 \leq k \leq r, 1 \leq l \leq t$, $Z_{i j}$ for $i \geq 0,1 \leq j \leq t+r$, or $X_{i}^{\prime}$ for $i \geq 0$ (see Section 3). Assume first that $M \cong U_{k l}, 1 \leq k \leq r, 1 \leq l \leq t$, where $t+r$ is the parameter of (ad 4). Then we have a monomorphism $Y_{l} \rightarrow U_{k l}$, which induces a monomorphism of functors $\gamma: \operatorname{Hom}_{\Lambda}\left(-, Y_{l}\right) \rightarrow \operatorname{Hom}_{\Lambda}\left(-, U_{k l}\right)$ and the set $\mathcal{S}_{\gamma}$ of all indecomposable modules 
$N$ such that coker $\gamma(N) \neq 0$ is finite. Indeed, $\mathcal{S}_{\gamma}=\left\{U_{i j} \mid 1 \leq i \leq k, 1 \leq j \leq l\right\}$. Again, coker $\gamma$ is finitely generated. Therefore, we get that coker $\gamma$ is of finite length and $T_{0} \operatorname{Hom}_{\Lambda}\left(-, Y_{l}\right) \cong T_{0} \operatorname{Hom}_{\Lambda}\left(-, U_{k l}\right)$. Assume now that $M \cong Z_{i j}, i \geq 0,1 \leq$ $j \leq t+r$. We consider two subcases.

- $\quad$ For $i \geq 0,1 \leq j \leq t$ we have monomorphisms $X_{i} \oplus U_{r j} \rightarrow Z_{i j}$ and $Y_{j} \rightarrow$ $U_{r j}$. Hence, by Lemma 2.2, we infer that $X_{i} \oplus Y_{j} \rightarrow Z_{i j}$ is a monomorphism. Again, we get the induced monomorphism of functors $\delta: \operatorname{Hom}_{\Lambda}\left(-, X_{i} \oplus Y_{j}\right) \rightarrow$ $\operatorname{Hom}_{\Lambda}\left(-, Z_{i j}\right), \mathcal{S}_{\delta}=\left\{U_{p l}, Z_{k l} \mid 1 \leq p \leq r, 1 \leq l \leq j, 0 \leq k \leq i\right\}$, and $T_{0} \operatorname{Hom}_{\Lambda}\left(-, X_{i} \oplus Y_{j}\right) \cong T_{0} \operatorname{Hom}_{\Lambda}\left(-, Z_{i j}\right)$.

- For $i \geq 0, t+1 \leq j \leq t+r$ we have a monomorphism $X_{i} \oplus U_{r j} \rightarrow Z_{i j}$, where $U_{r, t+1}, U_{r, t+2}, \ldots, U_{r, t+r}$ are given indecomposable injective $T_{r}(k)$-modules. Again, we get the induced monomorphism of functors $\varepsilon: \operatorname{Hom}_{\Lambda}\left(-, X_{i} \oplus U_{r j}\right) \rightarrow$ $\operatorname{Hom}_{\Lambda}\left(-, Z_{i j}\right), \mathcal{S}_{\varepsilon}=\left\{U_{r, t+1}, \ldots, U_{r, t+j}, Z_{k l} \mid 0 \leq k \leq i, 1 \leq l \leq j\right\}$, and $T_{0} \operatorname{Hom}_{\Lambda}\left(-, X_{i} \oplus U_{r j}\right) \cong T_{0} \operatorname{Hom}_{\Lambda}\left(-, Z_{i j}\right)$.

Finally, assume that $M \cong X_{i}^{\prime}, i \geq 0$. Again, we have an obvious monomorphism $X_{i} \rightarrow X_{i}^{\prime}$, the induced monomorphism of functors $\zeta: \operatorname{Hom}_{\Lambda}\left(-, X_{i}\right) \rightarrow$ $\operatorname{Hom}_{\Lambda}\left(-, X_{i}^{\prime}\right), \mathcal{S}_{\zeta}=\left\{X_{k}^{\prime}, Z_{k l} \mid 0 \leq k \leq i, 1 \leq l \leq t+r\right\}$, and $T_{0} \operatorname{Hom}_{\Lambda}\left(-, X_{i}\right) \cong T_{0} \operatorname{Hom}_{\Lambda}\left(-, X_{i}^{\prime}\right)$.

(c) $\Lambda$ is a modified algebra of $A^{(l)}$ obtained by applying the admissible operation of type (ad 5). Since in the definition of ( $\operatorname{ad} 5)$ we use the finite versions (fad 1), (fad 2), (fad 3), (fad 4) of the admissible operations ( $\operatorname{ad} 1),(\operatorname{ad} 2),(\operatorname{ad} 3),(\operatorname{ad} 4)$ and the admissible operation ( $\mathrm{ad} 4$ ), we conclude that the required statements follows from the above considerations.

Therefore, we may assume that $M$ is in fact an $A^{(l)}$-module. Let $F=\operatorname{Hom}_{\Lambda}(-, M)$ and $G=\left.\operatorname{Hom}_{\Lambda}(-, M)\right|_{\bmod A^{(l)}}$. Let $I$ be the simple $\Lambda$-module corresponding to the extension vertex of $A^{(l)}[X]$, where $X$ is the pivot of the suitable admissible operation. Since $\operatorname{Hom}_{\Lambda}(I, M)=0$ then for any $A$-module $Z$ we get $F(Z)=G\left(Z^{\prime}\right)$, where $Z^{\prime}$ is the restriction of $Z$ to $A^{(l)}$. Moreover, the category $\bmod A^{(l)}$ is contained in the obvious way into the category $\bmod \Lambda$. From this we conclude that, if $T_{1}^{\prime} G=0$, then $T_{1} F=0$, where

$$
T_{1}^{\prime}: \mathcal{F}\left(\bmod A^{(l)}\right) \rightarrow \mathcal{F}\left(\bmod A^{(l)}\right) / \mathcal{F}_{1}\left(\bmod A^{(l)}\right)
$$

and

$$
T_{1}: \mathcal{F}(\bmod \Lambda) \rightarrow \mathcal{F}(\bmod \Lambda) / \mathcal{F}_{1}(\bmod \Lambda)
$$

are the canonical quotient functors. By [11] we have $T_{1}^{\prime} G=0$, and hence $T_{1} F=0$. Since $F$ is not of finite length, we get that $T_{0} F \neq 0$.

Let $\mathcal{X}$ be the full subcategory of $\bmod \Lambda$ generated by all indecomposable modules from the generalized multicoils and the postprojective components, and let $\mathcal{Y}$ be the full subcategory of $\bmod \Lambda$ generated by all indecomposable modules from the preinjective components. Note that, since the projective cover of any finitely presented functor is a functor $\operatorname{Hom}_{\Lambda}(-, N)$ for some module $N$, it is enough to check only Hom-functors. Therefore, by the above arguments we have $\mathrm{K}-\operatorname{dim} \mathcal{X}=1$. Moreover, using Lemma 2.1 we get that $\mathrm{K}-\operatorname{dim} \mathcal{Y}=2$. Hence, applying [11, Theorem 2.6] we obtain

$$
\mathrm{K}-\operatorname{dim}(\bmod \Lambda)=\max (\mathrm{K}-\operatorname{dim} \mathcal{X}+1, \mathrm{~K}-\operatorname{dim} \mathcal{Y})=2 .
$$

Finally, we can complete the proof by an obvious induction on the number of admissible operations leading from $A^{(l)}$ to $A$. 


\section{Concluding Remarks}

Since the tame quasitilted algebras of canonical type form a distinguished special class of tame generalized multicoil algebras, we obtain the following fact.

Corollary 5.1 Let $A$ be a tame quasitilted algebra of canonical type. The following statements are equivalent:

(i) $\mathrm{K}-\operatorname{dim}(\bmod A)=2$.

(ii) $\mathrm{K}-\operatorname{dim}(\bmod A)$ exists.

(iii) $A$ is domestic.

Let $A$ be an algebra. Recall that a cycle in a module category $\bmod A$ is a sequence $X_{0} \stackrel{f_{1}}{\longrightarrow} X_{1} \rightarrow \cdots \rightarrow X_{r-1} \stackrel{f_{r}}{\longrightarrow} X_{r}=X_{0}$ of nonzero nonisomorphisms in ind $A$, and the cycle is said to be finite if $f_{i} \notin \operatorname{rad}^{\infty}(\bmod A)$ for any $1 \leq i \leq r$. If every cycle in $\bmod A$ is finite then $A$ is said to be cycle-finite. Recall also that a component $\mathcal{C}$ of $\Gamma_{A}$ is called semiregular if $\mathcal{C}$ does not contain both a projective and an injective module. It has been proved in [15] that a semiregular component $\mathcal{C}$ of $\Gamma_{A}$ contains an oriented cycle if and only if $\mathcal{C}$ is a ray tube or coray tube (see remarks after definitions of admissible operations).

As an immediate consequence of Corollary 5.1 and [31, Theorem 5.1] we obtain the following fact.

Corollary 5.2 Let $A$ be a cycle-finite algebra such that every component of $\Gamma_{A}$ is semiregular, and $p d_{A} X \leq 1$ or id ${ }_{A} X \leq 1$ for all but finitely many isomorphism classes of modules $X$ in ind $A$. Then the following statements are equivalent:

(i) $\mathrm{K}-\operatorname{dim}(\bmod A)=2$.

(ii) $\mathrm{K}-\operatorname{dim}(\bmod A)$ exists.

(iii) A is domestic.

Open Access This article is distributed under the terms of the Creative Commons Attribution License which permits any use, distribution, and reproduction in any medium, provided the original author(s) and the source are credited.

Acknowledgments This work was supported by the research grant DEC-2011/02/A/ST1/00216 of the Polish National Science Center.

\section{References}

1. Assem, I., Simson, D., Skowroński, A.: Elements of the Representation Theory of Associative Algebras 1: Techniques of Representation Theory, London Math. Soc. Student Texts 65. Cambridge University Press, Cambridge (2006)

2. Assem, I., Skowroński, A.: Minimal representation-infinite coil algebras. Manuscripta Math. 67, 305-331 (1990)

3. Assem, I., Skowroński, A.: Multicoil algebras. In: Representations of Algebras, CMS Conf. Proc. vol. 14, pp. 29-68. Amer. Math. Soc., Providence (1993)

4. Assem, I., Skowroński, A., Tomé, B.: Coil enlargements of algebras. Tsukuba J. Math. 19, 453-479 (1995)

5. Auslander, M.: Representation theory of Artin algebras II. Commun. Algebra 1, 269-310 (1974)

6. Baer, D.: Homological properties of wild hereditary artin algebras. In: Lecture Notes in Math. vol. 1177, pp. 1-12. Springer, Berlin (1986) 
7. Bongartz, K., Gabriel, P.: Covering spaces in representation theory. Invent. Math. 65, 331-378 (1974)

8. D’Este, G., Ringel, C.M.: Coherent tubes. J. Algebra 87, 150-201 (1984)

9. Drozd, Y.A.: Tame and wild matrix problems. In: Lecture Notes in Math. vol. 832, pp. 242-258. Springer, Berlin (1980)

10. Geigle, W.: The Krull-Gabriel dimension of the representation theory of a tame hereditary Artin algebra and applications to the structure of exact sequences. Manuscripta Math. 54, 83-106 (1985)

11. Geigle, W.: Krull Dimension and Artin Algebras. In: Lecture Notes in Math. vol. 1177, pp. 135-155. Springer, Berlin (1986)

12. Kerner, O.: Tilting wild algebras. J. London Math. Soc. 39, 29-47 (1989)

13. Lenzing, H., de la Peña, J.A.: Concealed-canonical algebras and separating tubular families. Proc. London. Math. Soc. 78, 513-540 (1999)

14. Lenzing, H., Skowroński, A.: Quasi-tilted algebras of canonical type. Colloq. Math. 71, 161-181 (1996)

15. Liu, S.: Semi-stable components of an Auslander-Reiten quiver. J. London Math. Soc. 47, 405-416 (1993)

16. Malicki, P.: Generalized coil enlargements of algebras. Colloq. Math. 76, 57-83 (1998)

17. Malicki, P.: Domestic generalized multicoil enlargements of concealed canonical algebras. J. Algebra Appl. 13, 1350105 (2014). 10 pp

18. Malicki, P., de la Peña, J.A., Skowroński, A.: Cycle-finite module categories. In: Algebras, Quivers and Representations - Abel Symposium 2011. Abel Symposia 8, pp. 209-252 (2013)

19. Malicki, P., de la Peña, J.A., Skowroński, A.: Finite cycles of indecomposable modules. J. Pure Appl. Algebra 219, 1761-1799 (2015)

20. Malicki, P., Skowroński, A.: Almost cyclic coherent components of an Auslander-Reiten quiver. J. Algebra 229, 695-749 (2000)

21. Malicki, P., Skowroński, A.: Algebras with separating almost cyclic coherent Auslander-Reiten components. J. Algebra 291, 208-237 (2005)

22. Malicki, P., Skowroński, A.: On the additive categories of generalized standard almost cyclic coherent Auslander-Reiten components. J. Algebra 316, 133-146 (2007)

23. Malicki, P., Skowroński, A.: Algebras with separating Auslander-Reiten components. In: Representations of Algebras and Related Topics, EMS Ser. Congr. Rep., Eur. Math. Soc. Zürich, pp. 251-353 (2011)

24. Malicki, P., Skowroński, A.: Hochschild cohomology of generalized multicoil algebras. Colloq. Math. 136, 231-254 (2014)

25. Reiten, I., Skowroński, A.: Characterizations of algebras with small homological dimensions. Adv. Math. 179, 122-154 (2003)

26. Ringel, C.M.: Tame Algebras and Integral Quadratic Forms. In: Lecture Notes in Math. vol. 1099 (1984)

27. Simson, D., Skowroński, A.: Elements of the Representation Theory of Associative Algebras 2: Tubes and Concealed Algebras of Euclidean Type, London Math. Soc. Student Texts 71. Cambridge University Press, Cambridge (2007)

28. Simson, D., Skowroński, A.: Elements of the Representation Theory of Associative Algebras 3 : Representation-Infinite Tilted Algebras, London Math. Soc. Student Texts 72. Cambridge University Press, Cambridge (2007)

29. Skowroński, A.: Cycle-finite algebras. J. Pure Appl. Algebra 103, 105-116 (1995)

30. Skowroński, A.: Tame quasi-tilted algebras. J. Algebra 203, 470-490 (1998)

31. Skowyrski, A.: Cycle-finite algebras with almost all indecomposable modules of projective or injective dimension at most one. Colloq. Math. 132, 239-270 (2013)

32. Wenderlich, M.: Krull Dimension of Strongly Simply Connected Algebras. Bull. Pol. Acad. Sci. Math. 44, 473-480 (1996) 